\title{
The evolving clinical, genetic and therapeutic landscape of multiple endocrine neoplasia type 2
}

\author{
Serisha Moodley ${ }^{1}$, Frank Weber ${ }^{2}$ and Lois M Mulligan' \\ 'Division of Cancer Biology and Genetics, Cancer Research Institute, and Department of Pathology and Molecular Medicine, Queen's University, \\ Kingston, Ontario, Canada \\ 2Department of General-, Visceral- and Transplantations Surgery, Division of Endocrine Surgery, Medical Faculty, University of Duisburg-Essen, \\ Duisburg, Germany \\ Correspondence should be addressed to L M Mulligan: mulligal@queensu.ca
}

This editorial accompanies a thematic review section on 25 Years of RET and MEN2. The guest editors for this section were Lois Mulligan and Frank Weber

The association of medullary thyroid carcinoma (MTC) and pheochromocytoma (PHEO) that we now recognize as multiple endocrine neoplasia type 2 (MEN2) (Fig. 1) was first reported by John Sipple in 1961 (Sipple 1961), but over 30 years passed before the cause of this inherited cancer syndrome was identified as mutations of the rearranged during transfection (RET) receptor tyrosine kinase (Donis-Keller et al. 1993, Mulligan et al. 1993). In the ensuing 25 years, RET mutation detection has changed our approaches to MEN2 risk prediction, diagnosis, management and treatment and has greatly improved disease outcomes and quality of life for patients and families with the disease. Moreover, advances in research and technology and better understanding of the disease are pushing the barrier toward earlier, less intensive, personalized care.

The identification of activating RET point mutations as the cause of MEN2 represented a novel paradigm for the origins of hereditary cancers, which had previously been exclusively linked to tumor suppressors. As MEN2 mutations activate or enhance RET kinase function, there were relatively few unique mutations (Fig. 2), which simplified detection and interpretation. This has led to our current abilities to predict disease course and optimize management based on the specific RET mutation observed (Wells et al. 2015).

The current outlook of MEN2 clinical management, treatment and quality of care

In 'Advances in the management of MEN2', Samuel Wells provides an historical overview of the clinical description of

(c) 2018 Society for Endocrinology Published by Bioscientifica Ltd. Printed in Great Britain
MEN2 with its associated phenotypes and the development of genetic testing for RET point mutations that has further defined the disease subtypes, MEN2A and MEN2B (Wells 2018). The review also provides an update on current standard-of-care management and treatment options in the context of these mutations, including recommendations on biochemical screening, early surgical intervention, chemotherapy and the future hope of cure or long-term stability of disease offered by new molecular targeted therapeutics (Wells 2018). While Wells' review focuses on MTC in MEN2, Carole Guerin and coworkers elaborate on differences in phenotype of MEN2-associated PHEO and hyperparathyroidism compared to non-MEN2 familial forms with a discussion on changes to the clinical management of these conditions to improve patient quality of life (Guerin et al. 2018). Frederic Castinetti and coworkers describe in detail the unique genetic and phenotypic manifestations of MEN2B, the most aggressive form of MEN2. In 'A comprehensive review of MEN2B' (Castinetti et al. 2018), they elaborate on the difficulties of early evaluations to recognize MEN2B and highlight current survival outcomes and optimal management for MEN2B patients.

Andreas Machens and Henning Dralle in 'Advances in risk-oriented surgery for multiple endocrine neoplasia type 2 ' dive into 25 years of longitudinal data on surgical treatment outcomes, within the framework of RET mutation genotype, to elaborate on the benefits and risks of current surgical interventions (Machens \& Dralle 2018). Looking forward, they discuss the synergistic value of RET mutation genotyping, coupled with cellular 


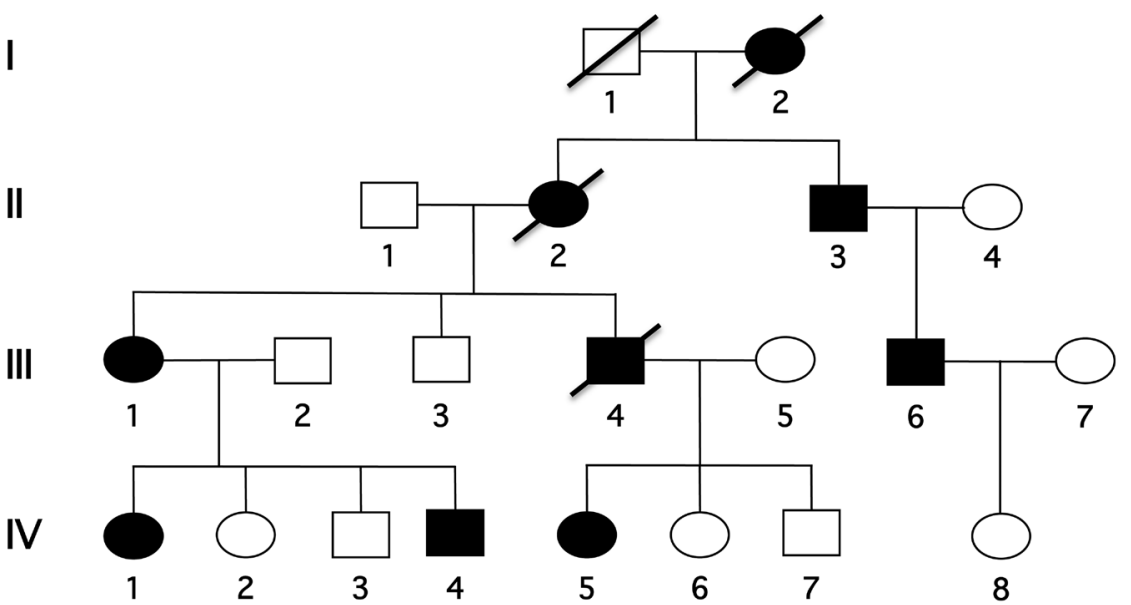

Figure 1

Pedigree of a family with MEN2 showing autosomal dominant inheritance pattern. biomarker screenings to determine the optimal surgical 'window of opportunity' to attenuate the potential risks associated with surgical treatments. In addition to surgical management, MEN2 has provided an ideal opportunity for precision medicine interventions using tyrosine kinase inhibitors to block RET activity. The current clinical use of previously developed and re-directed smallmolecule multi-kinase inhibitors, such as vandetanib and cabozantinib, in patients with advanced or metastatic MTC, has prolonged progression-free survival and stable disease, most notably for patients with the more aggressive MEN2B phenotype (Redaelli et al. 2018, Wells 2018). Yet, the poor efficacy and safety risks associated with off-target effects of these therapeutics leave much to be improved. In 'Novel targeted therapeutics for MEN2', Sara Redaelli and coworkers discuss the burgeoning growth in development or repositioning of novel RET-specific smallmolecule therapeutics currently in preclinical testing or early clinical trials (Redaelli et al. 2018). They also provide us with a glimpse of new drugs on the horizon from recent patent filings and discuss the development of combinatorial therapeutic strategies using standard chemotherapy with tyrosine kinase inhibitors or the addition of these inhibitors as second- or even third-line therapies in RET-associated cancers.

In 'Patient quality of life and prognosis in multiple endocrine neoplasia type 2', Joanna Grey and Kym Winter offer us a thoughtful and personal view of how these changes in our ability to manage MEN2 are affecting patients, their families and their care providers (Grey \& Winter 2018). They provide fascinating insight on the breadth of symptoms experienced by MEN2 patients and offer recommendations to better address patient needs by improving access to psychosocial support with molecular testing and clinical management to improve overall MEN2 patient health.

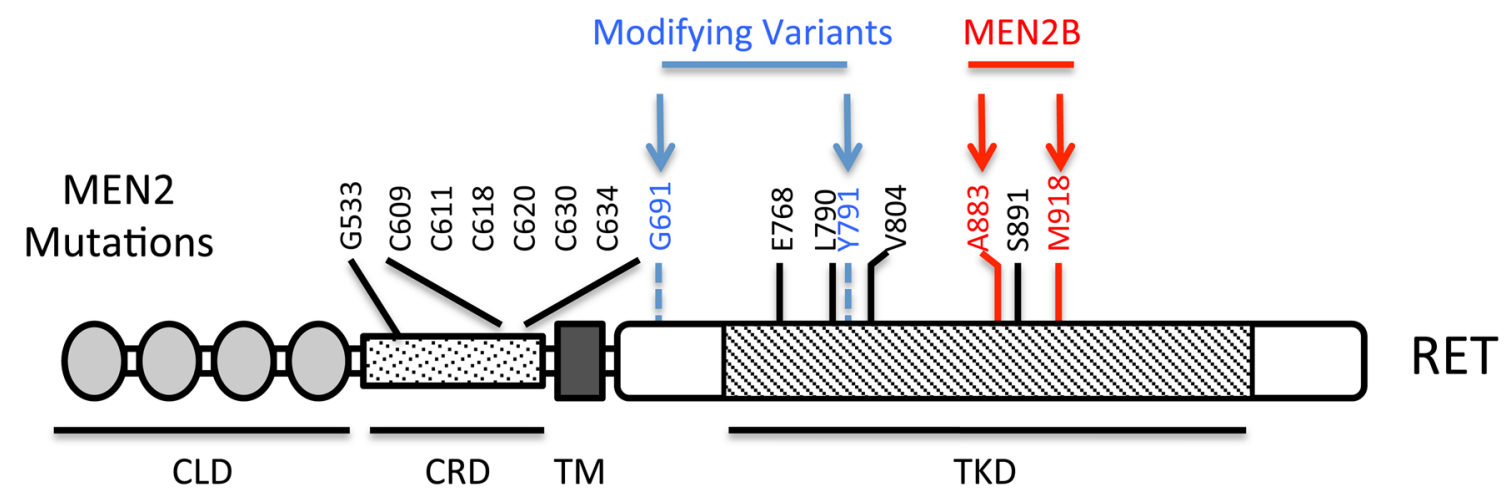

Figure 2

MEN2 mutations in the RET receptor tyrosine kinase. Schematic diagram showing locations of the common RET mutations identified in MEN2 patients. The two mutations associated with MEN2B are indicated in red. Two sequence variants suggested to act as modifiers, either affecting severity of other RET mutations or as risk-associated alleles in other cancers, are indicted in blue. RET protein domains indicated are CLD, cadherin-like domains; CRD, cysteine-rich domain; KD, kinase domain; TM, transmembrane domain. 


\section{Perspectives from the bench}

Molecular and genetic analyses have identified both activating and inactivating RET mutations associated with a range of human pathologies (Mulligan 2014). In 'Structure and function of RET in multiple endocrine neoplasia type 2', Ivan Plaza-Menacho elegantly details advances in RET structural biology that have provided insight into the molecular mechanisms of action of these mutations and how they may contribute to aberrant RET function in MEN2 and other RET-associated diseases (PlazaMenacho 2018). The current structural and molecular understanding of the RET ATP-binding and kinase domains and RET ligand-binding sequences, as well as the complexes formed by RET interactions with its ligands and co-receptors are of particular interest as we look to the next generation of RET-targeted therapeutics including small-molecule peptide mimetic inhibitors and synthetic blocking antibodies. Moving to preclinical evaluations, powerful murine models for RET have shown us how RET contributes to tumorigenesis and have been valuable tools in characterizing and validating RET-targeted agents (Wiedemann \& Pellegata 2016, Vitale et al. 2017). However, Tirtha Das and Ross Cagan also make a strong plea for the complementary value of 'Non-mammalian models of multiple endocrine neoplasia type 2', and their contribution to the understanding of RET function (Das \& Cagan 2018). They provide a comprehensive discussion of the use of zebrafish and drosophila models to dissect RET signaling pathways and as important in vivo tools for screening novel therapeutics and studying their efficacy and mechanism of action.

While research and therapeutic strategies have historically focused on the cancer cell itself, the review by Maria Castellone and Rosa Melillo beautifully describes how MEN2 RET mutations can influence the behavior of tumor-associated stromal and immune cells that, in turn, can modulate the behavior of the tumour (Castellone \& Melillo 2018). In 'RET-mediated modulation of tumor microenvironment and immune response in multiple endocrine neoplasia type 2', they dissect the complex mixture of cellular and non-cellular components that make up the MTC tumor microenvironment. They provide insight into the transcriptional programs and secretory responses that contribute to recruitment of stromal and immune cells and de-differentiation of cells for tumor tissue remodeling and highlight how these influence tumor-associated inflammation and cancer cell epithelial-to-mesenchymal transition that in turn promotes tumor spread. As the promise of immunotherapy for many cancers becomes recognized, the use of combination therapeutic options, including RET-targeted inhibitors coupled to immune checkpoint modulators, has exciting potential as future treatment for patients with advanced MTC.

\section{The net widens: RET in other pathologies}

RET's role in MTC has been well described in the past 25 years. However, as new technologies and screening methods improve our abilities to look more deeply into the genome, the contributions of RET to other pathologies are emerging. RET gene rearrangements that result in a constitutively active kinase have been well characterized in papillary thyroid carcinoma, and more recently in lung and peritoneal tumors (Flavin et al. 2009, Romei et al. 2016). Similar RET rearrangements have been found, albeit less frequently, in colon tumors and chronic myelomonocytic leukemia, while increased RET expression in general is associated with malignant phenotypes in a variety of cancers, including carcinomas of the pancreas and breast (Mulligan 2014, Romei et al. 2016). These findings have greatly expanded the patient populations that may benefit from RET-targeted therapies, and preclinical and early clinical trials of RET inhibition in these contexts are already underway (Redaelli et al. 2018).

RET loss of function mutations are found in familial forms of Hirschsprung disease, a congenital disorder of gut development (Plaza-Menacho 2018) and in patients with congenital abnormalities of the kidney and urinary tract (CAKUT) (Chatterjee et al. 2012). Recent research has also suggested that loss of RET-mediated signaling is linked to neuron degeneration and may play a role in late-onset Parkinson's disease (Drinkut et al. 2016). Together, these disparate roles suggests that use of targeted interventions for RET may need to be carefully modulated to maximize therapeutic value while minimizing the deleterious effects of RET inhibition on normal neural tissues (Requejo et al. 2017).

\section{The next 25 years}

It has been 25 years since the identification of RET mutations in MEN2. This issue touches on some of the many advances in understanding of the mechanisms of RET-mediated oncogenesis, which have led to enormous improvements in management of the disease, in essentially one generation. MEN2 truly represents a paradigm for precision medicine; changing patient care from the genetics of detection, through the use of 
biomarkers to identify optimal surgical windows, to the potential of RET-targeted therapies. We now have many more options than were thought possible in 1993, and greater hope for the future for patients and families with MEN2. As Andreas Machens and Henning Dralle predict in their review: 'The sweeping changes in the management of MEN 2 since the new millennium hold the hope that death and major morbidity from this uncommon disease, can be eliminated in our lifetime' (Machens \& Dralle 2018).

\section{Declaration of interest}

The authors declare that there is no conflict of interest that could be perceived as prejudicing the impartiality of this editorial.

\section{Funding}

This work did not receive any specific grant from any funding agency in the public, commercial or not-for-profit sector.

\section{References}

Castellone MD \& Melillo RM 2018 RET-mediated modulation of tumour microenvironment and immune response in multiple endocrine neoplasia type 2 (MEN2). Endocrine-Related Cancer 25 T105-T119. (https://doi.org/10.1530/ERC-17-0303)

Castinetti F, Moley J, Mulligan L \& Waguespack SG 2018 A comprehensive review on MEN2B. Endocrine-Related Cancer 25 T29-T39. (https://doi.org/10.1530/ERC-17-0209)

Chatterjee R, Ramos E, Hoffman M, VanWinkle J, Martin DR, Davis TK, Hoshi M, Hmiel SP, Beck A, Hruska K, et al. 2012 Traditional and targeted exome sequencing reveals common, rare and novel functional deleterious variants in RET-signaling complex in a cohort of living US patients with urinary tract malformations. Human Genetics 131 1725-1738. (https://doi.org/10.1007/s00439. 012-1181-3)

Das TK \& Cagan RL 2018 Non-mammalian models of multiple endocrine neoplasia type 2. Endocrine-Related Cancer 25 T91-T104. (https://doi.org/10.1530/ERC-17-0411)

Donis-Keller H, Dou S, Chi D, Carlson KM, Toshima K, Lairmore TC, Howe JR, Moley JF, Goodfellow P \& Wells SA 1993 Mutations in the RET proto-oncogene are associated with MEN 2A and FMTC. Human Molecular Genetics 2 851-856. (https://doi.org/10.1093/hmg/2.7.851)

Drinkut A, Tillack K, Meka DP, Schulz JB, Kugler S \& Kramer ER 2016 Ret is essential to mediate GDNF's neuroprotective and neuroregenerative effect in a Parkinson disease mouse model. Cell Death and Disease 7 e2359. (https://doi.org/10.1038/cddis.2016.263)
Flavin R, Jackl G, Finn S, Smyth P, Ring M, O’Regan E, Cahill S, Unger K, Denning K, Jinghuan L, et al. 2009 RET/PTC rearrangement occurring in primary peritoneal carcinoma. International Journal of Surgical Pathology 17 187-197. (https://doi. $\operatorname{org} / 10.1177 / 1066896908329593)$

Grey J \& Winter K 2018 Patient quality of life and prognosis in multiple endocrine neoplasia type 2. Endocrine-Related Cancer 25 T69-T77. (https://doi.org/10.1530/ERC-17-0335)

Guerin C, Romanet P, Taieb D, Brue T, Lacroix A, Sebag F, Barlier A \& Castinetti F 2018 Looking beyond the thyroid: advances in the understanding of pheochromocytoma and hyperparathyroidism phenotypes in MEN2 and of non-MEN2 familial forms. EndocrineRelated Cancer 25 T15-T28. (https://doi.org/10.1530/ERC-17-0266)

Machens A \& Dralle H 2018 Advances in risk-oriented surgery for multiple endocrine neoplasia type 2. Endocrine-Related Cancer $\mathbf{2 5}$ T41-T52. (https://doi.org/10.1530/ERC-17-0202)

Mulligan LM 2014 RET revisited: expanding the oncogenic portfolio. Nature Reviews Cancer 14 173-186. (https://doi.org/10.1038/nrc3680)

Mulligan LM, Kwok JBJ, Healey CS, Elsdon MJ, Eng C, Gardner E, Love DR, Mole SE, Moore JK, Papi L, et al. 1993 Germ-line mutations of the RET proto-oncogene in multiple endocrine neoplasia type $2 \mathrm{~A}$. Nature 363 458-460. (https://doi.org/10.1038/363458a0)

Plaza-Menacho I 2018 Structure and function of RET in multiple endocrine neoplasia type 2. Endocrine-Related Cancer 25 T79-T90. (https://doi.org/10.1530/ERC-17-0354)

Redaelli S, Plaza-Manacho I \& Mologni L 2018 Novel targeted therapeutics for MEN2. Endocrine-Related Cancer 25 T53-T68. (https://doi.org/10.1530/ERC-17-0297)

Requejo C, Ruiz-Ortega JA, Bengoetxea H, Bulnes S, Ugedo L \& Lafuente JV 2017 Deleterious effects of VEGFR2 and RET inhibition in a preclinical model of Parkinson's disease. Molecular Neurobiology [epub]. (https://doi.org/10.1007/s12035-017-0733-x)

Romei C, Ciampi R \& Elisei R 2016 A comprehensive overview of the role of the RET proto-oncogene in thyroid carcinoma. Nature Reviews Endocrinology 12 192-202. (https://doi.org/10.1038/nrendo.2016.11)

Sipple JH 1961 The association of pheochromocytoma with carcinoma of the thyroid gland. American Journal of Medicine 31 163-166. (https://doi.org/10.1016/0002-9343(61)90234-0)

Vitale G, Gaudenzi G, Circelli L, Manzoni MF, Bassi A, Fioritti N, Faggiano A, Colao A \& Group N 2017 Animal models of medullary thyroid cancer: state of the art and view to the future. EndocrineRelated Cancer 24 R1-R12. (https://doi.org/10.1530/ERC-16-0399)

Wells SA Jr 2018 Advances in the management of MEN2: from improved surgical and medical treatment to novel kinase inhibitors. EndocrineRelated Cancer 25 T1-T13. (https://doi.org/10.1530/ERC-17-0325)

Wells SA Jr, Asa SL, Dralle H, Elisei R, Evans DB, Gagel RF, Lee N, Machens A, Moley JF, Pacini F, et al. 2015 Revised American Thyroid Association guidelines for the management of medullary thyroid carcinoma. Thyroid 25 567-610. (https://doi.org/10.1089/ thy.2014.0335)

Wiedemann T \& Pellegata NS 2016 Animal models of multiple endocrine neoplasia. Molecular and Cellular Endocrinology 421 49-59. (https://doi.org/10.1016/j.mce.2015.07.004)

Received in final form 1 November 2018

Accepted 7 November 2018

Accepted preprint published online 7 November 2018 (c) 2018 Society for Endocrinology Published by Bioscientifica Ltd. Printed in Great Britain 